\title{
3D Visualisation for Education, Diagnosis and Treatment of lliotibial Band Syndrome
}

\author{
Erin Beveridge \\ Digital Design Studio \\ Glasgow School of Art \\ Glasgow, UK \\ erinbeveridge@ hotmail.com
}

\author{
Minhua Ma \\ Digital Design Studio \\ Glasgow School of Art \\ Glasgow, UK \\ m.ma@gsa.ac.uk
}

\author{
Paul Rea \\ School of Life Sciences \\ University of Glasgow \\ Glasgow, UK \\ paul.rea@glasgow.ac.uk
}

\author{
Kim Bale \\ Digital Design Studio \\ Glasgow School of Art \\ Glasgow, UK \\ k.bale@gsa.ac.uk
}

\author{
Paul Anderson \\ Digital Design Studio \\ Glasgow School of Art \\ Glasgow, UK \\ p.anderson@gsa.ac.uk
}

\begin{abstract}
The evolution of medical imaging technologies and computer graphics is leading to dramatic improvements for medical training, diagnosis and treatment, and patient understanding. This paper discusses how volumetric visualization and 3D scanning can be integrated with cadaveric dissection to deliver benefits in the key areas of clinicianpatient communication and medical education. The specific area of medical application is a prevalent musculoskeletal disorder-iliotibial (IT) band syndrome. By combining knowledge from cadaveric dissection and volumetric visualization, a virtual laboratory was created using the Unity 3D game engine, as an interactive education tool for use in various settings. The system is designed to improve the experience of clinicians who had commented that their earlier training would have been enhanced by key features of the system, including accurate three-dimensional models generated from computed tomography, high resolution cryosection images of the Visible Human dataset, and surface anatomy generated from a white light scan of an athlete. The finding from the virtual laboratory concept is that knowledge gained through dissection helps enhance the value of the model by incorporating more detail of the distal attachments of the IT band. Experienced clinicians who regularly treat IT band syndrome were excited by the potential of the model and keen to make suggestions for future enhancement.
\end{abstract}

Keywords-volumetric visualization, $3 D$ reconstruction, iliotibial band syndrome, anatomy education.

\section{INTRODUCTION}

IT band syndrome is one of the leading causes of lateral knee pain affecting runners and cyclists. Athletes with this condition typically require treatment involving several stages, one of which is a muscle-strengthening programme. Athlete compliance is a key requirement and it will influence the treatment outcome. For athletes a basic understanding of the underlying biomechanics and anatomy of their medical condition improves recovery and helps prevent a recurrence of the injury [6]. Therefore, rehabilitation can be viewed as particularly important and makes understanding what lies behind it an important area of study. Rehabilitation requires anatomical knowledge and clinical diagnosis necessitates the application of this knowledge to surface anatomy because it is the form in which the condition is presented to the clinician. Therefore, development of educational 3D models must also incorporate $3 \mathrm{D}$ surface anatomy.

Lower limb anatomy is of great clinical interest as understanding of this forms the basis of treatment for many musculoskeletal conditions. The argument for introducing $3 \mathrm{D}$ models into undergraduate physiotherapy education is to consolidate a three dimensional appreciation of the anatomy that is absent due to the absence of cadaveric dissection in the curriculum. To accurately represent this mode of learning, dissection of this region was performed on two cadavers to gain the knowledge of the relevant anatomy and first-hand experience of the spatial relationships to be recreated. The dissection also gives insight into structures that are not well represented in 2D imaging, such as the fascia and specifically the IT band. By applying traditional methods and capitalizing on modern technologies, the value of this model is elevated.

The project includes development of an interactive 3D model created from volumetric data and 3D scanning and a number of detailed interviews of physiotherapists and distillation of feedback from those practicing in the field of musculoskeletal disorders. The first step provides practitioners with an interactive, anatomically accurate 3D reconstruction of the lower limb to allow them to explore the spatial anatomy, and to virtually deconstruct the muscle relationships of importance in IT band syndrome. The interviews evaluate the experiences of the participants using the model and establish their views on the usefulness of the model as a training aid.

\section{MEDICAL IMAGING ADVANCES}

Although diagnostics is a common application of imaging in medicine, imaging is being extended to embrace new areas such as education, pre-operative planning, treatment and inter-disciplinary communication.

Anatomy is arguably one subject ideally positioned to adapt and exploit new developments with 3D technology. Here the concept of spatial relations is imperative. Being able to view in $3 \mathrm{D}$ gives much more clarity to a variety of users whereas, historically, conventional CT and MRI data is read by specialists who, through years of training, are able to assimilate a 3D mental image from 2D slices. Restricting this skill to these specialists creates barriers thereby limiting communication to both clinicians and patients.

Many major scanning manufacturers [7] now incorporate 3D image-generating software into their products. The process of generating 3D images from these diagnostic modalities is not fully automated, giving rise to certain challenges. Mathematic algorithms are the driving force behind generating the $3 \mathrm{D}$ images from a collection of $2 \mathrm{D}$ 
data. Certain challenges have been overcome, such as automating the segmentation of bony structures. However, delineating softer tissues such as muscle still presents a great challenge and is an active area of research [8]. If the deliverable of automatic segmentation of soft tissues is met, the application of this software in radiology departments could become routine.

\section{A. The Visible Human Dataset}

Many advances, including the Visible Human dataset, contribute to the increased understanding of anatomy and function of the human body. The dataset provides the most complete volumetric dataset available, with MRI, CT and cryosection photographs for normal male and female anatomy at $1 \mathrm{~mm}$ and $0.33 \mathrm{~mm}$ intervals respectively [12]. Following the impact of this dataset, similar projects spawned to represent different populations, e.g. the Chinese Visible Human project in 2002. Creation of more recent databases has seen improvements in methodology, by developing a low temperature laboratory the preservation of more delicate structures such as teeth and blood vessels has been more successful. The potential of such databases as an anatomical platform is ever increasing due to such advances. However, there are some structures, such as the fascia, which cannot be reconstructed accurately from this data, consequently cadaveric dissection remains highly valid.

\section{B. White Light Scanning}

The aforementioned advances in medical imaging have revolutionized the ability to see internal body structures. In diagnosis the surface of the body can be just as important. White light scanning technology is exploring applications in medicine already routinely used in other fields. Computed triangulation forms the basis of this technology: a grid pattern is projected onto surfaces with calculations taking into consideration the position of camera, projector and surface to be scanned [2] to produce a point cloud. A post process triangulation algorithm then produces a highly accurate representation of a surface [14]. Being noninvasive and non-contact this approach is appealing when applied in burns therapy.

The application of white light scanning technology is also currently being explored in the development of prostheses as translated scan data is immediately usable in computer aided drawing software, improving the speed of development of the prosthesis. As the price of the white light scanning technology declines, more affordable applications will emerge in concert with rapid prototyping, especially the potential applications in pre-operative planning.

\section{Recent Changes in Anatomy Curriculum}

Anatomical knowledge is required in all healthcare professions and its use starts at the point of initial patient examination to aid diagnosis, treatment and patient-clinician communication. The method for acquiring this knowledge in a capacity which promotes understanding is a demanding task. Dissection is undisputed as a powerful method that allows direct interaction and provides a visuospatial grasp of anatomy. Despite this, the majority of UK undergraduate physiotherapy courses do not include handling of cadaveric material.

Although hailed as the most effective method of anatomy teaching, dissection of cadavers has its limitations. They are not representational in terms of colour, texture or form relative to live bodies. This would seem to suggest a role for visualization technology because it can be representative of the live body in a knowledge-based capacity. This technology would be particularly beneficial in healthcare professions where cadaveric dissection is desirable but is either too costly or not presently on the curriculum.

Surface anatomy as a subject area has seen little innovation, yet as far as anatomy education goes this external investigation is possibly the oldest modality. It bridges the gap between underlying anatomy knowledge and clinical presentation of many conditions. Surface anatomy teaching incorporates the use of a living body with the aim of transfer of knowledge from associated cadaveric dissection. However, with advances in medical technology means there is great potential for educational enhancement here.

\section{ILIOTIBIAL BAND SYNDROME}

IT band syndrome is an overuse injury that occurs in response to repetitive motion, and it is one of the leading causes of knee pain in cyclists and the primary cause of lateral knee pain in runners. Participation in sports where IT band syndrome is prevalent has increased. Running and cycling participation in Europe is on a rising trend.

The approach taken by professional athletes training for events requiring endurance necessitates an understanding of the biomechanics of the body. Great physical demands are placed on them and they must plan accordingly, whereas the preparation stages are often overlooked by amateur athletes. Often those competing for the first time underestimate the need to add mileage at a steady rate to allow the body time to adapt and recover. Without this preparation additional demands are placed on their body which cannot be supported. As a result of this inexperience and lack of knowledge, IT band syndrome is a musculoskeletal disorder which is regularly seen by healthcare professionals. Clinicians are required to find a way to effectively educate them about the condition and the necessary rehabilitation programme required to treat the condition.

\section{A. Anatomy of the Iliotibial Band}

The IT band is a continuation of the facsia lata, encompasses the entire thigh, and is an area gaining interest into the causation of muscle pain. Given the increasing trend in favour of fascia treatments, there is a pressing need to improve understanding of the concept. Currently diagnostic imaging techniques cannot depict this structure in a comprehensible manner for patients or clinicians alike. Similarly, existing 3D models do not incorporate this structure. Specifically the representation of the IT band in models does not highlight its attachments. This is critically important for understanding the associated symptoms and biomechanics related to the condition, which 
this study is focused on, especially since the distal attachment is where symptoms present most severely.

As IT band syndrome was originally depicted as a friction syndrome [4] it was postulated that a bursa may exist beneath the IT band at the lateral femoral epicondyle, and so was considered a potential source of inflammation and consequential pain. However more recent studies, illustrate the presence of adipose tissue at the point of inflammation [4] [9]. Histological studies of tissue reveal the presence of Pacinian corpuscles [4]. These mechanoreceptors respond to transient shifts in pressure, with a particularly specialized ability to sense vibrations, making them highly susceptible to rapid changes in joint positions [1]. This may be an underlying contributing factor to pain felt at this region in people suffering from IT band syndrome.

Moving proximally, from the knee to the hip, the IT band is a thickening of facsia lata extending and covering the tensor facsia latae (TFL) and lateral aspect of the thigh. The deeper layers of this portion attach and blend with the lateral intermuscular septum.

The relationship of the IT band with the TFL has been extensively studied to determine the actions and roles of each as separate entities [6]. Thus proper representation of this relationship must also be accurately depicted in a model. Hip musculature plays an important role in IT band syndrome so the spatial relationships must be closely considered. The most proximal attachment of the IT band is at the bony hip where the tendon of gluteus maximus is adjacent, thus it may also have a role to play in IT band syndrome [4].

To create a model aiding understanding of IT band syndrome, it must incorporate the important aspects of its anatomy, specifically its attachments which to date are poorly demonstrated in similar models. Recreating the spatial relationship between relevant muscles is also critical in aiding understanding of the underlying biomechanical factors, which have to be addressed in treatment.

\section{B. Diagnosis of IT Band Syndrome}

As an overuse injury, continued exercise induces pain. Persistence leads to an inability to perform the desired physical activity such as running or cycling. Overuse injuries cause damage at a greater rate than the body can respond and repair due to repetitive motions at the joints [10]. The body's ability to adapt and repair biological tissues in response to stress is based on Wolff's law-a phenomenon which is a time constrained process and inadequate recovery will lead to the development of an overuse injury [5]. As an exercise induced condition the pain generally subsides upon cessation of the aggravating movements [6]. However, amongst athletes and people looking to keep fit there is a natural predisposition to exercise even when pain is being experienced hence the recurring nature of the condition.

Clinical examination and case history build enough evidence to confidently diagnose this condition. Pain can be reproduced for diagnostic purposes by performing the Noble test, which involves applying pressure to the site of pain during which time the patient is in a supine position with their leg flexed at 30 degrees [9]. This degree of flexion is considered the impingement zone and has implications for athletes.

\section{MATERIALS AND Methods}

\section{A. Materials}

Two cadavers were provided from the Laboratory of Human Anatomy at University of Glasgow, licensed under the Human Tissue (Scotland) 2006 and Anatomy Act 1984. The cadavers ( 86 and 101 years old) had been preserved following the University standard embalming protocol before the study commenced. No lower limb pathologies were present. The following tools were used for dissection; a size 4 Swann Morton scalpel handle, a size 24 scalpel blade and a pair of blunt forceps. A 12.1 megapixel digital camera was used for documentation.

The participant for white light scanning was a 30 -year old female triathlete who exercises at a competitive level. Participants for semi-structured interviews included a recently graduated physiotherapist working within the NHS. a physiotherapist within private practice who holds an undergraduate degree in sports therapy and physiotherapy and has extensive experience working with athletes as well as NHS experience; a physiotherapist in private practice specialising in sports injuries; and a sports massage therapist and personal trainer, currently studying for a degree in sports and exercise science at university.

Visible Human dataset: male, 38-year old, died from lethal injection [12]. CT and high-resolution cryoslice images from this dataset were used. The following software was used: Amira 5.0, Autodesk Maya 2011, Pixologic Z Brush 4, Unity 3 Version 3.4.1, Adobe Photoshop CS5 and Adobe After Effects. The Artec White Light Scanner was used for the surface scan. It captures data in 24-bit colour to $0.1 \mathrm{~mm}$ of accuracy.

\section{B. Methods \\ 1) Cadaveric dissection}

The same protocol was followed for both cadavers. In the supine position a longitudinal superficial incision was made proximally from the iliac crest to the region of the tibial tuberosity distally. Proximal and distal transverse incisions allowed reflection of the skin and subsequent exposure of the subcutaneous fat. Following imaging of the subcutaneous fat, it was removed to the level of the deep fascia. The fascia was imaged then carefully removed to leave intact its lateral thickening, the iliotibial band. In preparation for imaging particular care was taken to clean up and expose the attachment sites of this, proximally at the iliac crest and distally at the lateral aspects of the femur and tibia. The iliotibial band was removed at the site of the lateral femoral condyle to expose any underlying adipose tissue.

\section{2) Surface anatomy model: white light scan}

The scanner is handheld and can freely move around the subject projecting the light pattern onto the surface of the skin. The scan was taken superiorly from just above the pelvis to the foot on the floor inferiorly. By moving the scanner around the subject changes in the light pattern are 
recognized by the software, and cloud point data is generated on the screen. The first scan was taken with the participant's legs in full extension in a fixed position. The participant was then asked to stand with knees flexed at approximately 30 degrees of flexion. The final position was a deeper squat, with knees flexed as far as was comfortable whilst scanning took place. Each position was scanned twice to obtain sufficient data.

Following scanning, the data from repeated scans was first aligned by selecting several recognizable points such as hip, knee and ankle. It generates point cloud data representative of the leg surface and translated into a threedimensional polygon mesh complete with high quality textures. This was exported as obj and jpeg files for further processing. The triangulated meshes create surfaces with a very high polygon count, which requires polygon reduction for user interaction in the Unity engine. The mesh was simplified by applying a decimation algorithm in Zbrush.

\section{3) 3D Reconstruction from Visible Human Dataset}

\section{Data acquisition}

To reconstruct the relevant bones the $\mathrm{CT}$ data was chosen and slices from the level of the third lumbar vertebrae superiorly to midway down the tibia and fibula inferiorly were selected. The axial CT images had a resolution of $512 \times 512$ and were taken at $1 \mathrm{~mm}$ intervals. The cryosection photographs were used for reconstruction of the muscles. Slices were chosen and scaled by half to give a more workable dataset. These cryosections were also obtained at $1 \mathrm{~mm}$ intervals, corresponding to the CT images. Each pixel in these images represented an area of $0.33 \mathrm{~mm}$. To provide an isotropic volume, the pixel size was reduced to $1 \mathrm{~mm}$, taking into account the number of images that had been loaded. It gave an import ratio of 1:1:6. Data was imported into Amira, with anatomical images converted to grayscale by selecting the correct channel.

\section{Segmentation and surface extraction}

Pre-processing of medical data typically includes applying a filtering step. However in this situation filtering led to the loss of muscle fibre detail which is useful for identifying muscle boundaries, so no filter was applied.

The process of segmentation largely took place in the axial plane with individual structures of interest being assigned to different user defined materials. One of the simplest forms of segmentation is a threshold-based approach applied to the CT data to delineate the bones. Muscles cannot be delineated by such an automatic process and for tissues of this nature a laborious semi-automatic process was required. Region growing was utilised for certain muscles whilst the close proximity of many muscles dictated manual segmentation of the 2D slices. Process speed was increased by an interpolation algorithm applied for every $5 \mathrm{~mm}$. Manual manipulation was applied to correct any defects.

Following segmentation, a 3D model was generated by applying a surface extraction algorithm to the contour sets. This produced surfaces with millions of polygons unsuitable for real time rendering. Each material was exported to allow further processing and with alternative modelling software.

\section{Mesh editing and rendering}

Following surface extraction, Autodesk Maya was used for refining the model as well as adding textures and lighting.
The polygonal meshes were cleaned up by removing nonmanifold geometry and a planar texture map was assigned to each muscle. UV mapping, in this case for meshes with millions of polygons, was too complex and time consuming so Maya shaders were used to create the desired look. Three-point lighting was used to create a scene that show cased the musculature and bone in a realistic manner. The scene was rendered using mental ray, with ray casting for the shadows and final gather enabled. Finally the models, similar to the surface scan, were decimated in Zbrush to reduce the polygon count and allow them to be imported into Unity.

\section{4) Creating an Interactive Application}

Four scenes were created to provide a start-up screen and three different game modes, one of which is presented in Figure 1. Two scenes utilized the anatomy model whilst the other focused on the surface anatomy. The features required throughout the applications were developed using Unity script. Movement was added to the main camera to allow panning and zooming, whilst the models themselves were given rotational movement both along the $\mathrm{x}$ and $\mathrm{y}$ axes via onscreen buttons. An additional button resets the original position and orientation of the model. A second, background camera allows images to be added giving added context to each scene. Each object displays its name on demand and instructions specific to each scene are displayed onscreen. Horizontal sliders for adjusting the transparency of individual game objects are provided for each object. To create the effect of virtual deconstruction in the lab, a dragand-drop script was added to allow movement of components around the scene. A rebuild button was added to restructure the model to its original state. Links created between each level and the start-up menu allows user navigation to unfold between the different scenes.

To incorporate the surface anatomy, a treatment clinic was created, demonstrating where symptoms present and thus providing a diagnostic reference tool. The virtual laboratory and classroom facilitate teaching and communication through encouraging exploration of spatial relationships and textual display.

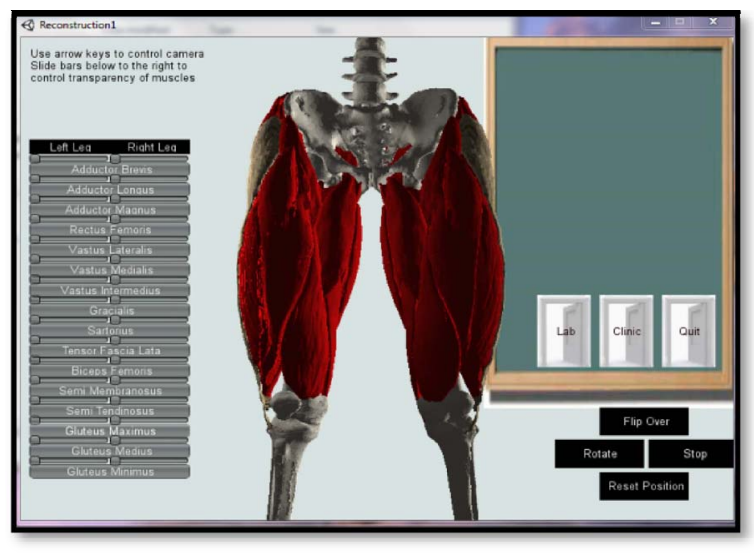

Figure 1: Interactive application: the classroom scene gives the users the opportunity to make muscles transparent to reveal what lies behind. A muscle's function, origin and insertion is displayed on the blackboard when selected. 


\section{RESULTS}

The dissection of the lateral thigh region allowed for clear examination of the IT band. Dissection uncovered its origin, positioning, insertion and spatial relationship to other muscles, which act on the hip and knee.

\section{A. $3 D$ Reconstruction from Visible Human Dataset}

Through the processes of segmentation and surface extraction a 3D model of lower limb musculoskeletal anatomy was created. The bones were easiest to identify and thus an isosurface was quickly constructed from the CT data. The muscles required more user interaction and anatomical knowledge. Table 1 shows all extracted structures. Most of the segmentation took place in the axial plane, with comparison of the full colour high-resolution cryosection images aiding this process. The final surfaces constructed accurately depict the anatomical details and correct spatial orientations that are necessary for understanding the underlying functional anatomy of IT band syndrome. Figure 2 gives static snapshots of the reconstructed limb from posterior superior and medial perspectives and Figure 3 shows screenshots of the short animation created to illustrate the main aspects of the condition and respective rehabilitation.

Table 1. Segmented structures from CT and cryosection images from the Visible Human Male dataset

\begin{tabular}{|l|l|}
\hline Pelvis, Femur, Tibia \& Fibula & Iliotibial Band \\
Vastus Intermedius & Vastus Medialis \\
Rectus Femoris & Vastus Lateralis \\
Semimembranosus & Semitendinosus \\
Biceps Femoris & Adductor Longus \\
Adductor Magnus & Adductor Brevis \\
Sartorius & Gracilis \\
Tensor Fascia Latae & Gluteus Maximus \\
Gluteus Minimus & Gluteus Medius \\
\hline
\end{tabular}
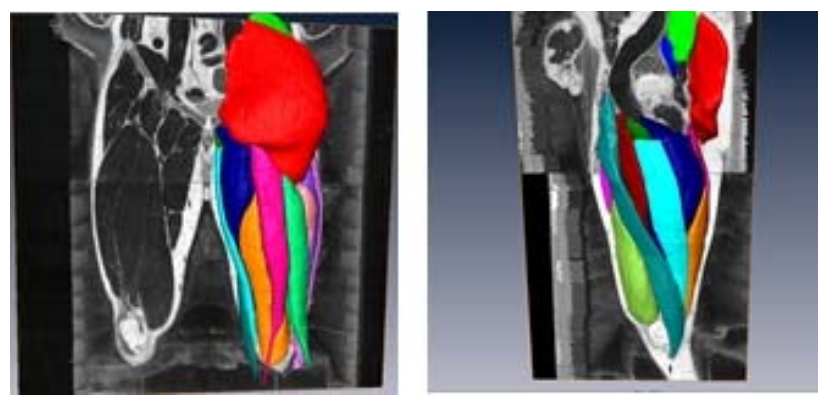

Figure 2: 3D reconstruction results. The muscles can be viewed in posterior superior (left) and medial (right) in concert with CT slices to validate the segmentation.
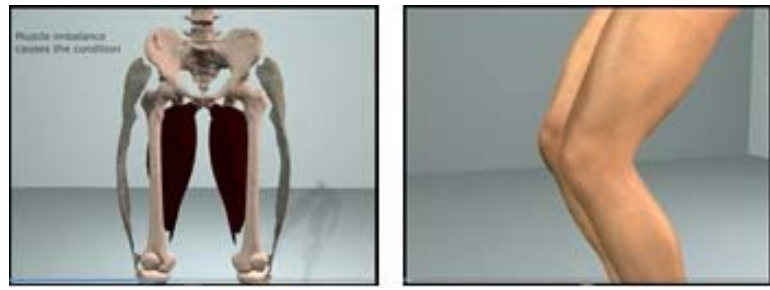

Figure 3: Reconstructed anatomy model (left) and the surface model (right) rendered using mental ray

\section{B. Evaluation}

Three of the four participants have a physiotherapy degree; for two their curriculum included cadaveric dissection. Both participants without experience of cadaveric dissection expressed strong opinions that exposure to the anatomy in three dimensions would have been a useful surrogate and would have consolidated their anatomy knowledge.

For all interviewed, anatomy teaching, aside from the dissection component, consisted of didactic lectures, practical surface techniques with textbooks for reference. The massage therapist supplemented her own learning by downloading several 3D applications, which weren't being provided or recommended by the teaching institution. Whilst no one felt their anatomy education was compromised, all could see potential for incorporation of $3 \mathrm{D}$ models to enhance the learning experience.

Patient compliance was unanimously considered to be the area deriving the greatest benefit from patient education. Patient education was a focus in all settings, from those working within the NHS to independent therapists working in private clinics. Where it perhaps divides is patient motivation and their desired involvement. As all physiotherapists interviewed worked at some point within the NHS and private practice, specifically with athletes, the consensus was that athletes were by far the most involved in the management of their treatment. To educate patients on their conditions and involve them in the treatment, all therapists used images in books, with the massage therapist using downloaded 3D applications. The physiotherapist working within the NHS felt they had limited access to resources to help in this process.

IT band syndrome is a condition that all therapists had regular exposure to and agreed the management of the condition was complex and required patient compliance. The benefits of the project application as a tool to aid education of this condition were demonstrated. The physiotherapists commented on the fact that during their training 3D visualization products, like the one demonstrated, were not available at that time.

The surface anatomy model received positive feedback. It was regarded as a beneficial reference resource, which could be used in clinical practice. One key suggestion was that a database containing multiple 3D surface anatomy models of different conditions would be useful. The reason was summed up by a participant: "getting exposure to all types of conditions in a realistic context just isn't possible prior to clinical practice, and 3D models will help remedy that weakness."

Several recurring themes introduced potential future developments. Identifying bony prominences and grouping muscles according to function were both regarded as being of benefit to an undergraduate educational package. Targeting postgraduate and specialist training programmes was also repeatedly raised. Possible targets include work based on the 'anatomy trains' and trigger point therapy. Another repeated point was the potential to adapt the $3 \mathrm{D}$ model to show pathology as well as normal anatomy. This would provide a direct comparison and would benefit patients and students. A specific suggestion in relation to IT band syndrome was that the model could be improved to 
include all of the lower limb anatomy which would then demonstrate the role of distal mechanics.

\section{Conclusions}

The dissection showed little scope for sheer movement of the IT band across the femoral epicondyle due to these resolute attachments, thus does not support proposed friction mechanism models. Instead, dissection supported the notion that compression would be a more plausible cause of inflammation here. This coupled with a suggestive role for atypical distal mechanics in this condition meant it was important to clearly demonstrate these attachments in the model. Similar models often overlook the importance of these attachments, especially on the tibia, which can be crucial for patient understanding.

A firm grasp of the relative anatomy was the perfect basis to develop a 3D model of this region. Manual segmentation of muscles from the Visible Human anatomical images was a time consuming exercise, but it allowed for anatomical knowledge to be applied to the development process ensuring accuracy. The IT band was particularly difficult to identify. Applying the knowledge acquired at the dissection stage and therefore being able to recognise the attachments gave much needed direction. This shows the potential exists for traditional dissection to complement the development of new models which have a pressing need to incorporate structures such as the fascia, which outwith a dissection laboratory is very difficult to identify.

IT band syndrome is exercise induced, and a condition mostly limited to those of a more athletic build. Demonstrating this accurately in dissection is difficult due to the largely elderly population of cadaveric specimens. Selecting the male dataset from the visible human potentially provides more accurate representation of the relevant anatomy (38-year old male).

Inclusion of the surface anatomy as a diagnostic reference tool is a feature unique to this model. Surface anatomy is fundamental in physiotherapy and its incorporation elevated the validity of the model as a training tool. The use of a white light scanner in obtaining this surface data was quick, easy and relatively cheap in comparison to alternatives, such as laser scanning. It is also less sensitive to movement than laser scanners, so a better alternative for use on living subjects.

The targets of improving patient-clinician communication and innovating healthcare training were assessed by professionals in the field. For IT band syndrome, it was concluded that high quality patient education leads to increased compliance. This in turn relates to an increase in the success of treatment. It was agreed by all participants that there is a pressing need for innovative tools to help them meet their patient education targets, which are under scrutiny due to budget constraints. There is particular scope for use of 3D communication tools in private clinics where many patients already pay for the opportunity to optimise their treatment. The validation process highlighted that the market is underpenetrated due to a general lack of awareness of what is available. One interviewee, who had looked into this area, was using 3D models in her personal practice with patients, showing there is demand when practitioners discover what is available.

\section{A. Limitations and Future Work}

The Visible human dataset has shortcomings due to the methodology involved in obtaining the images. For the cryosections created, the cadaver had to be segmented into four blocks, resulting in a loss of data at certain intervals, including regions in this reconstruction [14]. However it was not felt to have impacted at the level of anatomy included for the purposes of study but is worth consideration if finer anatomical structures were to be incorporated.

The model and its various applications provide a solid framework for further development to incorporate different aspects of musculoskeletal anatomy, conditions and treatments. As an educational tool further detail such as grouping muscles by functional territories and visualizing the ligaments and tendons would consolidate its application.

At the present a large body of research into treatments and underlying mechanisms is currently turning to the fascia. Developing a model that can incorporate the fascia to give it representation would be of interest to physiotherapists. However, like dissection of fascia in cadavers, the ability to segment this structure accurately is a substantial task. This project demonstrates that the combination of traditional methods with modern technology may provide a route for optimising the visualization of difficult to grasp concepts and deliver them in an accessible format to patients and clinicians alike. Treatment driven applications are likely to hold greater merit in a commercial market because they confer tangible benefits to both clinician and patient.

\section{REFERENCES}

[1] Brisben, A., Hsiao, s., \& AO, J. (1999). Detection of Vibration Transmitted Through an Object Grasped in the Hand. The American Physiological Society, 81 (4), 1548-1558.

[2] D'Apuzzo, N. (2006). Overview of 3D surface digitization technologies in Europe. San Jose: SPIE.

[3] Fairclough, J., Hayashi, K., Toumi, H., Lyons, K., Bydder, G., Phillips, $\mathrm{N}$., et al. (2007). Is iliotibial band syndrome really a friction syndrome. Journal of Science and Medicine in Sport , 10, 74-76.

[4] Fairclough, J., Hayashi, K., Toumi, H., Lyons, K., Bydder, G., Phillips, N., et al. (2006). The functional anatomy of the iliotibial band during flexion and extension of the knee: implications for understanding iliotibial band syndrome. Journal of Anatomy , 208, 309-316.

[5] Hreljac, A. (2004). Impact and Overuse Injuries in Runners. Medicine and Science in Sports and Exercise, 845-849.

[6] Khaund, R., \& Flynn, S. (2005). Iliotibial Band Syndrome: A Common Source of Knee Pain. American Family Physician, 71 (8), 1545-1550.

[7] Liang, G.Y., Fang, B., Wang, Y., Wu, L., \& Chen, P. (2011). Design Of An Interactive 3D Medical Visualisation System. IEEE.

[8] Ma, Z., Manuel, J., Tavares, R., \& Jorge, R. (2008). Segmentation of structures in 2D medical images. Venice: 5 th. European Congress on Computational Methods in Applied Sciences and Engineering.

[9] Muhle, M., Ahn, J., Yeh, L., Bergman, G., RD, B., Schweltzer, M., et al. (1999). Iliotibial Band Friction Syndrome: MR Imaging Findings in 16 Patients and MR Arthrographic Study of Six Cadaveric Knees. Radiology , 212 (1), 103-110.

[10] Renne, J. (1975). The Iliotibial Band Friction Syndrome. The Journal of Bone and Joint Surgery, 57-A (8), 1110-1111.

[11] Sakas, G. (2002). Trends in medical imaging: from 2D to 3D. Computers and Graphics , 26 (4), 577-587.

[12] Spitzer, V., Ackerman, A., Scherzinger, A., \& Whitlock, D. (1996). The Visible Human Male: A Technical Report. Journal of American Medical Informatics Association, 3 (2), 118-130.

[13] Stecco, C., Porzionato, A., Lancerotto, L., Stecco, A., Macchi, V., Day, J., et al. (2008). Histological study of the deep fasciae of the limbs. Journal of Bodywork and Movement Therapies, 12, 225-230.

[14] Treleaven, P., \& Wells, J. (2007). 3D Body Scanning and Healthcare Applications. IEEE Computer Society. 